\title{
Structural Persistence of Three Dimensional Autonomous Formations
}

\author{
Changbin $\mathrm{Yu}^{1}$, Julien M. Hendrickx ${ }^{2},{\text { Barış } \text { Fidan }^{1} \text {, Brian D.O. Anderson }}^{1}$ \\ ${ }^{1}$ National ICT Australia Ltd. and \\ Research School of Information Sciences \& Engineering, the Australian National University, \\ 216 Northbourne Ave., Canberra ACT 2601 Australia \\ $<$ brian.anderson, baris.fidan, brad.yu>@nicta.com.au*** \\ 2 Department of Mathematical Engineering, Université Catholique de Louvain, \\ Avenue Georges Lemaitre 4, B-1348 Louvain-la-Neuve, Belgium \\ Hendrickx@inma.ucl.ac.be ${ }^{\dagger}$
}

\begin{abstract}
Built upon a recently developed theoretical framework, we consider some practical issues raised in multi-agent formation control in three dimensional space. We introduce the partial equilibrium problem, which is associated with unsafe control of a formation in practical 3-dimensional applications. We define structurally persistent graphs, a class of persistent graphs free of any partial equilibrium problem. In real deployment of control of multi-agent systems, formations with underlying structurally persistent graphs are of interest. We study the connections between the allocation of degrees of freedom (DOFs) across agents and the characteristics of persistence and/or structural persistence of a directed graph. We also show how to transfer degrees of freedom between agents, when the formation changes with new agent(s) added, to preserve persistence and/or structural persistence.
\end{abstract}

\section{INTRODUCTION}

In [1], we have generalized the definition of persistence to $\Re^{d}$ for $d \geq 3$, seeking to provide a theoretical framework for real world applications, which often are in 3dimensional space as opposed to the plane. We also have derived some new properties of persistent graphs and given an operational criterion to determine if a graph is persistent. In this paper, we demonstrate that a persistent formation, as defined in [1], may also

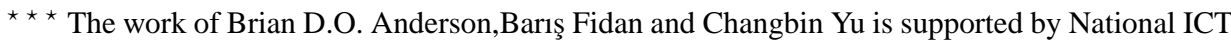
Australia, which is funded by the Australian Government's Department of Communications, Information Technology and the Arts and the Australian Research Council through the Backing Australia's Ability Initiative. Changbin Yu is an Australia-Asia Scholar supported by the Australian Government's Department of Education, Science and Training through Endeavours Programs.

$\dagger$ The work of Julien M. Hendrickx is supported by the Belgian Programme on Interuniversity Attraction Poles initiated by the Belgian Federal Science Policy Office, and by the Concerted Research Action (ARC) "Large Graphs and Networks" of the French Community of Belgium. The scientific responsibility rests with its authors. Julien M. Hendrickx is a FNRS (Belgian Fund for Scientific Research) fellow. 
suffer from a practical problem where each agent can move to a position which satisfies the constraints on it once all the other agents are fixed but it is not possible to satisfy all the constraints on all the agents at the same time.

In Section 2, we formally characterize the above problem, which we call the partial equilibrium problem, and which is closely associated with unsafe control of a formation in practical 3-dimensional applications. We then introduce the definition of a structurally persistent graph, a class of persistent graphs free of any partial equilibrium problem. In real deployment of control of multi-agent system, formations with underlying structurally persistent graphs are of interest. It is established in Secction 2 incidentally that in two dimensions, structural persistence and persistence are equivalent.

In Section 3, we focus on the connections between allocation of degrees of freedom (DOFs) across agents and the characteristics of persistence and/or structural persistence of a directed graph. We also show how to transfer degrees of freedom between agents, when the formation changes with new agent(s) added, to preserve persistence and/or structural persistence. We study cycle-free graphs in $\Re^{3}$ and show some more powerful results that exist in this special case, such as the existence of a quadratic time criterion to verify the cycle-free property and to decide persistence, which automatically guarantees structural persistence.

We end the paper with concluding remarks in Section 4. Note that all the proofs are omitted due to space limitations. However, a full version of this work together with the campanion paper [1] is available in preprint from the authors.

\section{PARTIAL EQUILIBRIUM PROBLEM AND STRUCTURALLY PERSISTENT GRAPHS}

Consider a persistent graph $G=(V, E)$ in $\Re^{d}(d \in\{2,3, \ldots\})$. The partial equilibrium problem we want to avoid is the following: There is a subset $\tilde{V} \subset V$ of vertices such that all the vertices in $\tilde{V}$ are at fitting positions whatever the positions of the vertices in $V \backslash \tilde{V}$ are, but there exists no position assignment for the vertices in $V \backslash \tilde{V}$ such that the whole representation is fitting. For example, consider the 3-dimensional persistent graph $\bar{G}$ shown in Figure 1, an associated set $\bar{d}$ of desired lengths $d_{i j}>0$ for all the edges $\overrightarrow{(i, j)}$, and a realization $\bar{p}$ of $\bar{d}$ in agreement with Figure 1 . Identify $\tilde{V}$ with $\{1,2\}$. Since the vertices 1 and 2 have zero out-degrees, they are at fitting positions for any representation of the graph, whatever the positions of 3, 4, 5 are. However, there are representations of $\bar{G}$ arbitrarily close to $\bar{p}$ where the vertices 3,4 , and 5 cannot be at fitting positions at the same time. From the perspective of formations, in the formation represented by $\bar{G}$, there exist two leaders, 1 and 2, which are allowed to move freely in $\Re^{3}$ without any constraint. This freedom, however, makes it impossible in some cases for the agents 3, 4, and 5 to meet all the distance constraints on them, although $\bar{G}$ is persistent, according to the definition given in Section 3 of [1]. In such a case, we will say that $\bar{G}$ is in partial equilibrium.

The existence of such partial equilibrium problems in three and higher dimensional spaces makes it necessary to analyse persistent graphs further and introduce new concepts such as structural persistence that will be defined in this section. In $\Re^{2}$, however, there is no persistent graph suffering from partial equilibrium problems, as ex- 


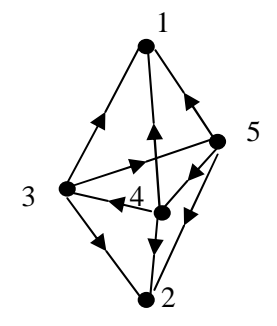

Fig. 1. A persistent graph in $\Re^{3}$ which is not structurally persistent.

plained later in Theorem 1. Let us consider a persistent graph $G=(V, E)$ in $\Re^{d}$ $(d \in\{2,3, \ldots\})$ with a representation $p$. Let $\bar{d}$ be the set of distances corresponding to $p . G$ is in partial equilibrium, and thus has the partial equilibrium problem if there exists a non-empty vertex subset $\tilde{V} \subset V$, a constant $\varepsilon>0$ and a mapping $p_{\bar{\varepsilon}}$ indexed by $\bar{\varepsilon},\left\{p_{\bar{\varepsilon}}: \tilde{V} \rightarrow \Re^{d} \mid 0<\bar{\varepsilon} \leq \varepsilon\right\}$ such that for any $\bar{\varepsilon} \leq \varepsilon$ the following hold:

1. $d\left(p(i), p_{\bar{\varepsilon}}(i)\right) \leq \bar{\varepsilon}, \forall i \in \tilde{V}$.

2. For all $i \in \tilde{V}, p_{\bar{\varepsilon}}(i)$ is a fitting position with respect to $\bar{d}$, irrespective of the positions of the vertices in $V \backslash \tilde{V}$.

3. There exist no fitting representation $p^{\prime}: V \rightarrow \Re^{d}$ in $B(p, \bar{\varepsilon}) \triangleq\left\{\bar{p}: V \rightarrow \Re^{d} \mid d(p, \bar{p}) \leq \bar{\varepsilon}\right\}$, with respect to $\bar{d}$, such that $p^{\prime}(i)=p_{\bar{\varepsilon}}(i), \forall i \in \tilde{V}$.

If a persistent graph is not in partial equilibrium, it is called a structurally persistent graph. To analyse this concept further, it is defined that for a given directed graph $G=$ $(V, E)$, a subgraph $G^{\prime}=\left(V^{\prime}, E^{\prime}\right)$ is a practically closed subgraph of $G$ if for any vertex $i \in V^{\prime}, d_{G^{\prime}}^{+}(i) \geq \min \left\{d, d_{G}^{+}(i)\right\}$, where $d_{G^{\prime}}^{+}(i)$ denotes the number of outgoing edges incident to the vertex $i$ of a graph $G^{\prime}$. We remark that a closed subgraph is always a practically closed subgraph, since each vertex of it satisfies the criterion defined above.

In the 2-dimensional example shown in Figure 2, where $V^{\prime}=\{1,2,3\}, G^{\prime}$ is a practically closed subgraph of $G$ but not a closed subgraph of $G$. All the outgoing edges of 1 and 2 in $G$ remain in the subgraph $G^{\prime}$. Vertex 3, on the other hand, has two outgoing edges in $G^{\prime}$ (making $G^{\prime}$ a practically closed subgraph) and another one not in $G^{\prime}$. From a perspective of formations where vertices denote agents and edges denote awareness, in $G$, although 3 is aware of 4 , it may not be able to react to correctly maintain its distance from 4 because its position is locked by the constraints with respect to the vertices 1 and 2 .

The relation between partial equilibrium problems and practically closed subgraphs is examined in the following propositions.

Proposition 1 Consider a persistent graph $G=(V, E)$ in $\Re^{d}(d \in\{2,3, \ldots\})$ with a representation $p$ and a set $\bar{d}$ of distances corresponding to $p$. Let $G^{\prime}=\left(V^{\prime}, E^{\prime}\right)$ be a subgraph of $G$ where $V^{\prime}$ is a non-empty vertex subset of $V$. Then, $G^{\prime}$ is a practically closed subgraph of $G$ if and only if there exist a constant $\varepsilon>0$ and a mapping $p_{\bar{\varepsilon}}$ indexed by $\bar{\varepsilon},\left\{p_{\bar{\varepsilon}}: V^{\prime} \rightarrow \Re^{d} \mid 0<\bar{\varepsilon} \leq \varepsilon, p_{\bar{\varepsilon}}\right.$ is the restriction of $\left.p \rightarrow V^{\prime}\right\}$ such that for any $\bar{\varepsilon} \leq \varepsilon$ the following hold: 

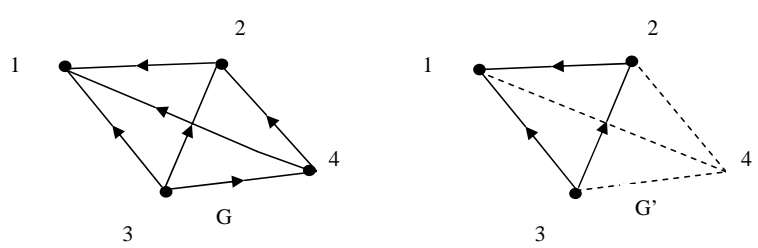

Fig. 2. A practically closed subgraph in $\Re^{2}$.

1. $d\left(p(i), p_{\bar{\varepsilon}}(i)\right) \leq \bar{\varepsilon}, \forall i \in V^{\prime}$, for all $p_{\bar{\varepsilon}}$ in the mapping set.

2. For all $i \in V^{\prime}, p_{\bar{\varepsilon}}(i)$ is a fitting position with respect to $\bar{d}$, irrespective of the positions of the vertices in $V \backslash V^{\prime}$.

Proposition 2 Consider a persistent graph $G=(V, E)$ in $\Re^{d}(d \in\{2,3, \ldots\})$ with a representation $p$ and a set $\bar{d}$ of distances corresponding to $p . G$ is structurally persistent if and only if every non-empty practically closed subgraph of $G$ is persistent.

The following theorem, which uses Proposition 2, states that there is no partial equilibrium problem in $\Re^{2}$, as mentioned in the beginning of the section.

Theorem 1 Any persistent graph $G \in \Re^{2}$ is structurally persistent and has all its practically closed subgraphs persistent.

Proposition 2 states that a graph is not structurally persistent if and only if it contains a practically closed subgraph that is not persistent. Development of this notion leads to the following proposition, which gives another necessary and sufficient condition for a persistent graph to be structurally persistent.

Proposition 3 Let $G=(V, E)$ be a persistent graph in $\Re^{d}$. G is structurally persistent if and only if there is no non-persistent closed subgraph of $G$ with less than d vertices.

The following corollary, which is a major result of the section, and which immediately follows from Proposition 3, gives a more explicit necessary and sufficient condition for 3-dimensional persistent graphs to be structurally persistent. It also gives more insight for the problem encountered in the example in Figure 1.

Corollary 1 A persistent graph $G=(V, E)$ in $\Re^{3}$ is structurally persistent if and only if there is at most one leader ${ }^{3}$ in $G$.

Remark 1 For some dimensions $d>3$, one can have a non-persistent closed subgraph with less than d vertices in a graph that has only one leader. An example in $\Re^{6}$ is shown in Figure 3. In general, in a given d-dimensional persistent graph, the presence of a non-persistent closed subgraph can be checked by looking only at the vertices with outdegree less than $d-1$, which are finite in number because of the bound on the total DOF count. 


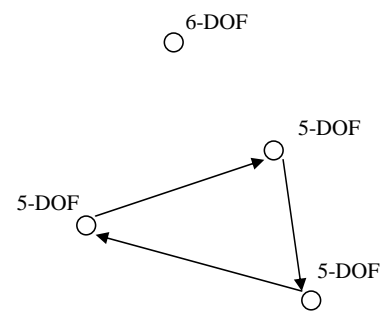

Fig. 3. A non-persistent closed subgraph in $\Re^{6}$ that does not have two leaders. Note that in $\Re^{6}$, the total DOF count of a persistent graph can be up to 21 .

Remark 2 In contrast to the case $d=3$, for $d \geq 4$, a non-persistent closed subgraph of a persistent graph can be connected. Consider, for example, the 4-dimensional directed graph $G=(V, E)$ shown in Figure $4 . G$ is constraint consistent because no vertex has an out-degree larger than 4. Moreover, it is minimally rigid and it can be obtained by removing an edge from the complete graph $K_{6}$, which is trivially rigid. On the other hand, the closed subgraph $G_{2}$ of $G$ is non-rigid and hence non-persistent. Therefore, $G$ is not structurally persistent. Note that $G_{2}$ is connected although it is non-persistent.

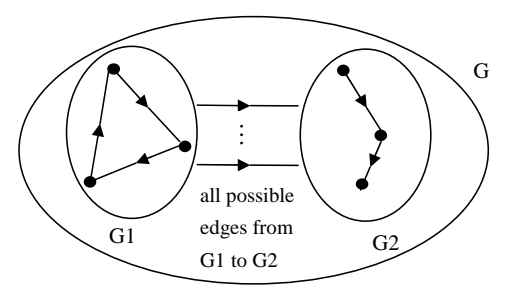

Fig. 4. A 4-dimensional persistent graph with a non-persistent and connected closed subgraph.

\section{ALLOCATION OF DOFs IN $\Re^{3}$ AND TRILATERATION}

In Section 2, we have seen that for a directed graph, persistence is not enough to avoid partial equilibrium problems. In the following subsections, we study how the way degrees of freedom happen to be allocated to the vertices of a directed graph is a key determinant of the structural persistence or otherwise of that graph. In an application scenario, this corresponds to giving/restricting the autonomy of certain agents (abstracted as degrees of freedom of vertices) of the formation [2].

\footnotetext{
${ }^{3}$ An agent is a leader if it has no constraints on its movement, e.g. in Figure 1, both agents represented by vertices 1 and 2 are leaders. In associated graphs, corresponding vertices have no outgoing edges.
} 


\subsection{DOF Allocation and Transfer via Directed Trilateration in $\Re^{3}$}

In this section, we study the properties of the directed version of Henneberg-like vertex addition in 3 and higher dimensions, which is an abstraction of the event that new agents join a formation, one at a time. We give examples of applying such operations to manipulate DOF allocation of persistent graphs, in particular, in $\Re^{3}$.

Let us consider a persistent graph $G=(V, E)$ in $\Re^{d}(d \in\{2,3, \ldots\})$ where $|V| \geq$ $d$. A directed $d$-vertex addition, DVA $(d, n)$ where $n \in\{0, \ldots, d\}$, transforms $G$ to another persistent graph $G^{\prime}=\left(V^{\prime}, E^{\prime}\right)$ where $V^{\prime}=V \cup\{i\}, E^{\prime}=E \cup\{\overrightarrow{(i, k)}$ : $\left.\left.\forall k \in V_{1}\right\} \cup \overrightarrow{(j, i)}: \forall j \in V_{2}\right\}, V_{1}, V_{2} \subseteq V, V_{1} \cap V_{2}=\emptyset,\left|V_{1}\right|=d-n,\left|V_{2}\right|=n$ , and $\operatorname{DOF}(j) \geq 1, \forall j \in V_{2},{ }^{4}$ provided that the vertices of $V_{1} \cup V_{2}$ do not lie in any $q$-dimensional hyperplane where $q<d$.

We note that from Lemma 2 of [1], constraint consistency is preserved with the directed $d$-vertex addition defined above. Moreover, from the following lemma which is drawn from $[3,4]$, we see that the rigidity is also preserved.

Lemma 1 [3, 4] A graph obtained by adding one vertex to a graph $G=(V, E)$ in $\Re^{d}$ and $d$ edges from this vertex to other vertices of $G$ is rigid if and only if $G$ is rigid.

Hence by Theorem 2 of [1], the graph obtained after applying a directed $d$-vertex addition on a persistent graph in $\Re^{d}$ is persistent, i.e., the $d$-directed vertex addition defined above preserves the persistence of the graphs.

Remark 3 Consider a persistent graph $G=(V, E)$ in $\Re^{d}$. Let $G^{\prime}=\left(V^{\prime}, E^{\prime}\right)$ be the graph obtained by applying the operation $D V A(d, n)$ to $G$, where $V^{\prime}=V \cup\{i\}$. Then we have: $D O F_{G^{\prime}}(i)=n ; D O F_{G^{\prime}}(j) \leq D O F_{G}(j), \forall j \in V$. and $D O F_{G}(j)-D O F_{G^{\prime}}(j) \in$ $\{0,1\}, \forall j \in V$.

In the remaining part of this section, we only consider $\Re^{3}$, although results can be easily expanded to higher dimensions. As a more convenient nomenclature in $\Re^{3}$, we use the term directed trilateration operation, abbreviated DT( $), \operatorname{DT}(n)$ in place of directed 3-vertex addition or DVA $(3, n)$.

An undirected graph formed by a sequence of trilateration operations starting with an initial undirected triangle, often called a trilateration graph, is guaranteed to be generically rigid in $\Re^{3}$ and globally rigid in $\Re^{2}$. A trilateration graph can always be constructed/deconstructed using a polynomial time algorithm, where a reverse trilateration can be performed by removing a vertex with degree 3 at each step. Note that a seed with 3 vertices is needed to initiate a trilateration sequence. However, two different directed triangular seeds can start a directed trilateration operation in $\Re^{3}$ as defined in Figure 5(a) and Figure 5(b) are called the leader-first follower-second follower ( $L-F F-S F$ ) and the balanced triangle $\left(B_{1} B_{2} B_{3}\right)$ seeds, respectively.

Remark 4 The leader-first follower-second follower seed is analogous to the leaderfollower structure defined for a 2-dimensional cycle-free graph [5]. The set of DOF counts of the seed vertices is $\{3,2,1\}$. The balanced triangle is nothing more than a directed triangle (cycle) in a cyclic graph and the corresponding DOF count set is $\{2$ $, 2,2\}$.

\footnotetext{
${ }^{4}$ Non-existence of $V_{2}$ means the corresponding $\operatorname{DVA}(n)$ cannot be performed for the graph.
} 


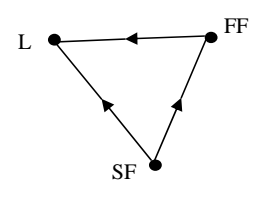

(a)

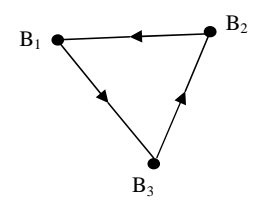

(b)

Fig. 5. The two directed triangular seeds.

Specifically in the application to 3 dimensional agent formations, note the meanings of the DT $(i)$ operation for different $i$ can be interpreted as follows,

- DT(3) means election of a new leader.

- DT(2) may result in either breaking/restoring the balanced control structure, or election of a new first-follower.

- DT(1) may also result in either breaking/restoring the balanced control structure at more a detailed level, or creation/change of second follower.

- DT(0) preserves the control structure and no decision has to be made by preexisting agents.

Noting that in a 3-dimensional persistent graph, there are at most 6 DOFs (as opposite to 3 DOFs in the $\Re^{2}$ case) to be allocated among the vertices, we can list the following six types of DOF allocation (abbreviated DOF allocation state $S_{1}$ to $S_{6}$ with DOF counts of vertices):

- $S_{1}=\{3,2,1,0,0, \ldots\}, S_{2}=\{2,2,2,0,0, \ldots\}$

$-S_{3}=\{3,1,1,1,0,0, \ldots\}, S_{4}=\{2,2,1,1,0,0, \ldots\}$

- $S_{5}=\{2,1,1,1,1,0,0, \ldots\}, S_{6}=\{1,1,1,1,1,1,0,0, \ldots\}$

Further, we define a transient type of DOF assignment $S_{0}=\{3,3,0,0, \ldots\}$, which can (only) be obtained by applying a DT(3) operation to $S_{3} . S_{0}$ is named "transient" because it apparently allows two leaders simultaneously in control of a formation, and hence this creates instability and we want the DOF assignment to avoid this state. Recall that the underlying directed graph of such a formation is a persistent graph with a partial equilibrium problem, i.e. it is persistent but NOT structurally persistent (An example of a graph that is in transient state $S_{0}$ can be seen from Figure 1).

We study the transformational relationship between the possible distribution of DOFs by applying the appropriate DT $(\cdot)$ operation using the "state transition diagram" shown in Figure 6. We have the following observations:

- Starting from any one of the two directed triangular seeds, we can build any graph with any of $S_{0}-S_{6}$ by adding at most three vertices using directed trilateration.

- Any desired DOF reallocation pattern(with no allocation to a specific vertex) can be achieved by at most four directed trilaterations starting with any of the six types of DOF allocation.

- Any desired stable DOF reallocation pattern can be achieved by at most three directed trilaterations starting with any stable DOF allocation. 


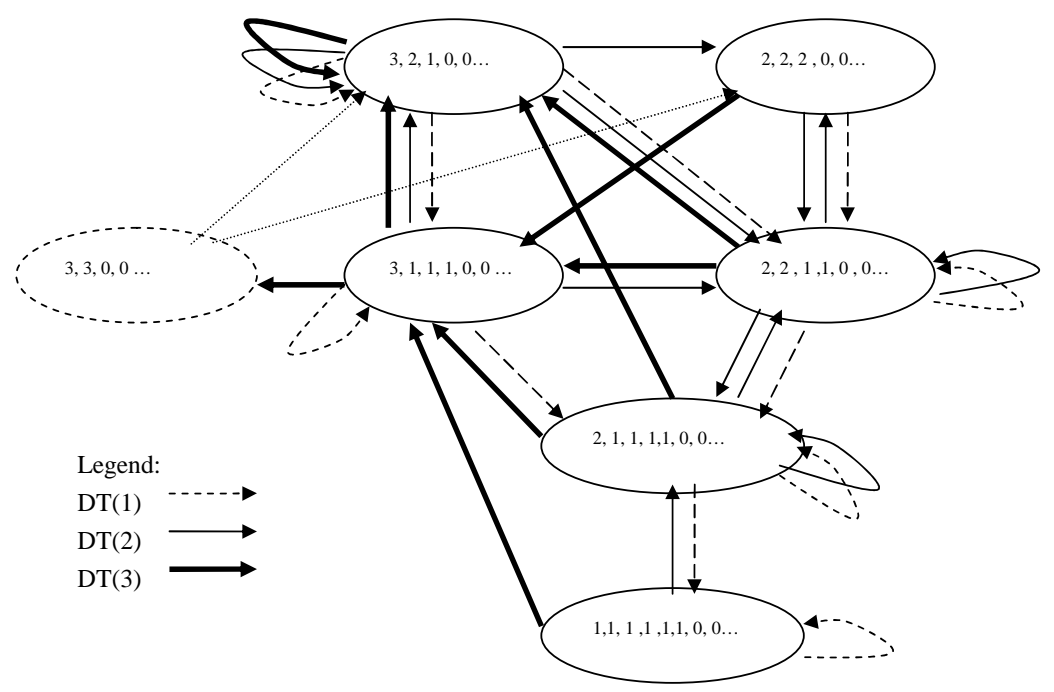

Fig. 6. The state transition diagram for directed trilaterations.

The observed results above gives an upper bound on the number of agents required in order to perform a system reconfiguration operation, such as replacement or elimination of leaders/first-follower/second-follower, or change to balanced cooperative control of 3 leaders. And it also gives the possible consequences in a closing ranks problem ${ }^{5}$, where the lost agent has a certain positive number of DOFs .

\subsection{Cycle-Free Graphs}

Persistence of cycle-free graphs in $\Re^{2}$ was studied in [5]. In this section, we derive a simple criterion to decide the persistence and structural persistence of cycle-free graphs in $\Re^{d}$. We also show an explicit way to build all the persistent cycle-free graphs.

Proposition 4 A graph obtained by adding one vertex to a graph $G=(V, E)$ in $\Re^{d}$ and at least $d$ edges leaving this vertex is persistent if and only if $G$ is persistent.

We thus know that a cycle-free graph obtained by successively adding vertices all with out-degree $d$, i.e. $\operatorname{DVA}(d, 0)$, to an initial seed of a cycle-free persistent graph containing only $d$ vertices is persistent.

Next,we focus on cycle-free persistent graphs in $\Re^{3}$, which have important application in safe control of multi-agent formations.

\footnotetext{
${ }^{5}$ The closing ranks problem for a given rigid formation which has lost a single agent, is to find new links between some agent pairs which, if maintained cause the resulting formation to again be rigid.
} 
Theorem 2 A cycle-free graph in $\Re^{3}$ having more than 2 vertices is persistent if and only if it has a closed subgraph which is the leader-first follower-second follower triangle, and every other vertex has an out-degree larger than or equal to 3.

Moreover, every cycle-free persistent graph in $\Re^{3}$ can be obtained from an original seed composed by the leader-first follower-second follower by adding vertices one by one in the way described in Proposition 4, i.e., each vertex is added with every incident edge outwardly directed.

We can also progressively deconstruct the cycle-free (persistent) graph by recursively removing one vertex at a time, where that vertex has at least 3 outgoing edges until we obtain the leader-first follower-second follower triangle. We conclude from these observations that the computational complexity of verifying both persistence and cycle-free properties of 3-dimensional graphs is quadratic in the number of vertices. In other word, if the deconstruction process cannot proceed for the graph, then the graph we are dealing with is not a persistent cycle-free graph. Note the cycle-free property allows only one leader in the graph, thus following Corollary 1, we have

Proposition 5 All cycle-free persistent graph in $\Re^{3}$ are also structurally persistent.

\section{CONCLUSION AND FURTHER WORKS}

In this paper, we considered some practical issues raised in multi-agent formation control in three dimensional space, building upon a recently developed theoretical framework. We introduced the partial equilibrium problem. We defined structurally persistent graphs, a class of persistent graphs free of any partial equilibrium problem, noting that for $d=2$, structural persistence is no different to persistence. We studied the connections between the allocation of degrees of freedom (DOFs) across agents and the characteristics of persistence and/or structural persistence of a directed graph. We proposed directed $d$-vertex addition operations for $\Re^{d}$. We also showed how to reallocate degrees of freedom between agents, when the formation changes with new agent(s) added, to preserve persistence and/or structural persistence. Finally, we gave some powerful results about cycle-free persistent graphs in $\Re^{3}$.

\section{References}

[1] J.M. Hendrickx, B. Fidan, C. Yu, B.D.O. Anderson, and V.D. Blondel. Rigidity and persistence of three and higher dimensional directed formations. To appear in the 1st International Workshop on Multi-Agent Robotic Systems-MARS05' as a companion of this paper.

[2] C. Yu, B. Fidan, and B.D.O. Anderson. Persistence acquisition and maintenance for autonomous formations. Submitted to the 2nd International Conference on Intelligent Sensors, Sensor Networks and Information Processing, 2005.

[3] W. Whiteley. Matroid Theory, volume 197 of Contemporary Mathematics, pages 171-311. American Mathemtical Society, 1996.

[4] W. Whiteley. Handbook of Discrete and Computational Geometry, chapter Rigidity and Scene Analysis, pages 893-916. CRC Press, 1997.

[5] J.M. Hendrickx, B.D.O. Anderson, V.D. Blondel, and J.-C. Delvenne. Directed graphs for the analysis of rigidity and persistence in autonomous agent systems. Submitted to the Int. J. Robust Nonlinear Control, 2005. 